\title{
Comparison of Morphology Evolution of Ge(001) Homoepitaxial Films Grown by Pulsed Laser Deposition and Molecular Beam Epitaxy
}

\section{Citation}

Byungha, Shin, John P. Leonard, James W. McCamy, and Michael J. Aziz. 2005. Comparison of morphology evolution of Ge(001) homoepitaxial films grown by pulsed laser deposition and molecular beam epitaxy. Applied Physics Letters 87(18): 181916.

\section{Published Version}

http://dx.doi.org/10.1063/1.2108115

\section{Permanent link}

http://nrs.harvard.edu/urn-3:HUL.InstRepos:2795957

\section{Terms of Use}

This article was downloaded from Harvard University's DASH repository, and is made available under the terms and conditions applicable to Other Posted Material, as set forth at http:// nrs.harvard.edu/urn-3:HUL.InstRepos:dash.current.terms-of-use\#LAA

\section{Share Your Story}

The Harvard community has made this article openly available.

Please share how this access benefits you. Submit a story.

\section{Accessibility}




\title{
Comparison of Morphology Evolution of Ge(001) Homoepitaxial Films Grown by Pulsed Laser Deposition and Molecular Beam Epitaxy
}

\author{
Byungha Shin, John P. Leonard*, James W. McCamy**, and Michael J. Aziz*** \\ Division of Engineering and Applied Sciences, Harvard University, Cambridge, MA \\ 02138 \\ Published as Applied Physics Letters 87, 181916 (2005). \\ This revised version, submitted August 22, 2005, \\ differs from final published version due to significant length cuts.
}

\begin{abstract}
Using a dual Molecular Beam Epitaxy (MBE)-Pulsed Laser Deposition (PLD) Ultra-High Vacuum chamber, we have conducted the first experiments under identical thermal, background, and surface preparation conditions to compare $\mathrm{Ge}(001)$ homoepitaxial growth morphology in PLD and MBE. We find that in PLD with low kinetic energy and in MBE the film morphology evolves in a similar fashion: initially irregularly shaped mounds form, followed by pyramidal mounds with edges of the square-base along $<100\rangle$ directions; the film roughness and mound separation increase with film thickness. In PLD with high kinetic energy, well-defined pyramidal mounds are not observed and the morphology rather resembles that of an ion-etched $\mathrm{Ge}(001)$ surface. The areal feature density is higher for PLD films than for MBE films grown at the same average growth rate and temperature. Furthermore, the dependence upon film thickness of roughness and feature separation differ for PLD and MBE. We attribute these differences to the higher yield of defect generation by energetic species in PLD.
\end{abstract}


While both Pulsed Laser Deposition (PLD) and Molecular Beam Epitaxy (MBE) are being widely used in growing high quality epitaxial films, there are distinct differences in these two techniques. In PLD the kinetic energy of incident species on a growing surface can be a few hundred $\mathrm{eV}$, in contrast to typical thermal evaporation energies of less than $1 \mathrm{eV}$ in MBE. PLD also occurs in short pulses resulting in an instantaneous flux many orders of magnitude higher than found in MBE at the same average deposition rate. These differences are expected to significantly affect kinetic processes during growth, the understanding of which has generated much interest and research activity for both fundamental and device-engineering purposes. There has accumulated a vast body of knowledge on kinetic processes in MBE. Particularly, in homoepitaxial film growth by MBE, there exists a well-known growth instability leading to mound formation due to an additional potential barrier for adatom transport at the downward step edge, the so-called Ehrlich-Schwoebel (ES) barrier. ${ }^{1,2}$ Experimental evidence of an instability induced by the ES barrier during MBE growth has been reported in many semiconductor materials including $\mathrm{Si}^{3}{ }^{3} \mathrm{Ge},{ }^{4,5}$ and $\mathrm{GaAs},{ }^{6}$ as well as in metals. $^{7}$ For example, in their study of homoepitaxial growth of $\mathrm{Ge}(001)$ by MBE, Van Nostrand et $a l .{ }^{4}$ experimentally determined the ES barrier by applying the model of Politi and Villain ${ }^{2}$ to their measurements of island density in sub-monolayer (ML) growth and of the interval before the first appearance of growth mounds.

Unlike in the case of MBE, the understanding of kinetic processes during PLD, despite its wide-spread usage in practical thin film growth over many years, ${ }^{8}$ is still immature. A question naturally arises: can we understand PLD in light of well-known MBE paradigm? There have been some theoretical ${ }^{9,10}$ and experimental ${ }^{11,12}$ studies comparing these two growth techniques in attempting to answer this question. The results of experimental studies are so sensitive to deposition conditions that it is crucial to compare the results from these two techniques under identical conditions ${ }^{12}$ : same temperature calibration, background pressure, sample preparation, etc. Kirschner and coworkers $^{11}$ demonstrated improved layer-by-layer growth of magnetic metals on $\mathrm{Cu}$ by PLD compared to thermal deposition. However, the results were limited to the first few ML of growth and the focus of the work was on the relationship between structure and magnetic properties. To gain more insight on kinetic processes during growth, it is desirable to examine the morphology of films beyond a few ML because kinetic processes often manifest themselves in an asymptotic limit. For example, the slope selection of the mound structure in $\mathrm{Fe}(001)$ MBE homoepitaxy was observed above 10ML. ${ }^{13}$ Hinnemann et al. ${ }^{9}$ performed kinetic Monte Carlo (KMC) simulations to compare PLD and MBE morphologies and concluded that the energetic nature of PLD must play a crucial role in determining the morphology of a system influenced by the ES barrier.

Here we report the results of a comparative study of homoepitaxial growth morphology of Ge(001) by PLD and MBE using a dual MBE-PLD ultra-high vacuum (UHV) chamber that provides the ability to conduct both experiments under identical conditions other than the different nature of the deposition flux in the two techniques. In particular, we address the effect of kinetic energy of incident species in PLD, which has not been well addressed previously in the growth regime influenced by the ES barrier.

Both MBE and PLD are conducted in the same UHV chamber with a base pressure of $7 \times 10^{-11}$ Torr. MBE is performed using a solid source Knudsen-type effusion 
cell. For PLD, a pulsed $\mathrm{KrF}^{+}$excimer laser of $248 \mathrm{~nm}$ wavelength ablates a rotating solid Ge target residing in the chamber. A pulse repetition rate of $20 \mathrm{~Hz}$ is used and a target-tosubstrate distance of $6 \mathrm{~cm}$. The fluence is varied between 4 and $7 \mathrm{~J} / \mathrm{cm}^{2}$ in order to tune the kinetic energy of depositing species. Ion probe measurements indicate an average kinetic energy of Ge ions during PLD using high fluence, $\sim 7 \mathrm{~J} / \mathrm{cm}^{2}$ and low fluence, $\sim 4$ $\mathrm{J} / \mathrm{cm}^{2}$ of $450 \mathrm{eV}$ and $300 \mathrm{eV}$, respectively. We refer to PLD using high fluence as PLD with high kinetic energy (PLD-HE) and PLD using low fluence as PLD with low kinetic energy (PLD-LE).

A substrate is cleaned by repeated rinsing with deionized water and exposure to ozone in order to grow a thick protecting oxide. ${ }^{14}$ After desorbing the oxide layer at $\sim 450{ }^{\circ} \mathrm{C}$ for 45 minutes, a (2X1) reconstruction on the surface is visible from Reflection High Energy Electron Diffraction (RHEED). A $30 \mathrm{~nm}$ buffer layer is grown at $370{ }^{\circ} \mathrm{C}$ by MBE, providing a clean starting surface with root-mean-square (rms) roughness less than $0.1 \mathrm{~nm}$. The growth temperature of both MBE and PLD films is $150{ }^{\circ} \mathrm{C}$. The deposition rate of MBE growth is $0.025 \mathrm{~nm} / \mathrm{s}$. In the case of PLD there is some variation in the deposition rate from run to run, $0.02-0.045 \mathrm{~nm} / \mathrm{s}$. Surface morphology is examined by $e x$ situ AFM in tapping mode on an area not irradiated by the RHEED beam. The film thickness is determined by Rutherford Backscattering Spectrometry (RBS) on a companion sample of Ge grown simultaneously on $\mathrm{Si}$.

In order to investigate morphological evolution AFM images of films at various thicknesses were collected. Representative images are shown in Fig. 1. As seen from Figs. 1(a)-1(c), during MBE the surface is initially smooth. As growth proceeds, irregularly shaped growth mounds form. Eventually they assume a well-defined pyramidal shape with the edges of the square-base along $\langle 100\rangle$ directions. An increase in mound separation with increasing film thickness is apparent from images, indicating coarsening of the mounds occurs during growth. Further deposition beyond the thickness of $205 \mathrm{~nm}$ (Fig. 1(c)) yields an amorphous surface film, indicating that epitaxial breakdown has occurred. The epitaxial thickness for MBE growth at $150{ }^{\circ} \mathrm{C}$ was determined to be $210 \mathrm{~nm}$ by a combination of an observation of amorphous rings in the RHEED pattern and RBS ion channeling data. Figure 1(c), in fact, is very close to the epitaxial breakdown threshold. In PLD-LE (middle column of Fig. 1), the surface morphology evolves in a similar fashion as in MBE. However, the areal mound density is higher for PLD films than for MBE films, which we attribute to the high instantaneous flux during PLD. We also note that in PLD-LE, extended epitaxial growth is possible, at least up to $410 \mathrm{~nm}$ as shown in Fig. 1(f), beyond the epitaxial thickness of MBE at the same growth temperature. The right column of Fig. 1 shows AFM images of the films grown by PLD-HE. Growth mounds are observed and they coarsen and roughen during deposition. However, unlike in MBE and PLD-LE, the growth mounds are not pyramidal for the range of thickness examined in this work, 49-278 nm. Instead they resemble features observed on the ion-etched Ge(001) surface; see, for example, Fig. 1(a) of Ref. ${ }^{15}$.

Suppressed corner diffusion has been proposed as a mechanism explaining the formation in epitaxial growth of pyramidal mounds with square bases with edges oriented $45^{\circ}$ from the directions of rapid adatom diffusion. ${ }^{16,17,18}$ The fact that pyramidal mounds are not observed in the case of PLD-HE suggests the possibility that corner diffusion may be enhanced by the high kinetic energy. The term "corner diffusion" in this context does not necessarily mean that atoms diffuse around a corner without losing contact with step 
edges. For example, adatoms either detached from or generated near a step can diffuse along a terrace around the corner, leading to effective corner diffusion. ${ }^{16}$

For a quantitative analysis of surface roughness, a plot of rms roughness vs film thickness is given in Fig. 2. We observe that PLD-HE generates the smoothest films among all three cases. Both MBE and PLD-LE have a similar rms roughness except that MBE films are not epitaxial beyond $210 \mathrm{~nm}$, whereas in PLD-LE epitaxial growth is possible at least up to twice this thickness. Improved epitaxial growth of $\mathrm{Si}(001)$ has been reported using energetic condensing particles with an average energy of 20 $\mathrm{eV}^{19,20}$ Collisionally enhanced filling of trenches between mounds, which act as nucleation sites of amorphous phase, has been suggested as a mechanism to explain the enhanced epitaxy. ${ }^{20}$ We believe that a similar mechanism is operating in PLD. However, it should be noted that the energy range of Ge atoms during PLD spans up to $1 \mathrm{keV}$ with an average around $300 \sim 450 \mathrm{eV}$, which is much larger than that used in previous studies employing ion beam sputter deposition. ${ }^{20}$ This energy range is well above bulk point defect creation energy of Ge but we see no sign of accumulated bulk point defects using RHEED and AFM, the techniques used in this study. Floro and co-workers ${ }^{21}$ demonstrated that bulk point defect diffusion can occur even at $-100{ }^{\circ} \mathrm{C}$. Therefore it is possible that point defects generated within the bulk migrate to the surface, becoming surface point defects, at our growth temperature of $150^{\circ} \mathrm{C}$. It has been shown that when the energy of particles bombarding a surface increases, the generation rate of defects, particularly of adatom-vacancy pairs, increases. For example, on the $\mathrm{Ge}(001)$ surface bombarded by $\mathrm{Xe}^{+}$ions the adatom yield increases an order of magnitude as the ion energy increases from 20 to $240 \mathrm{eV}^{22}$ Also molecular dynamics (MD) simulations of hyperthermal homoepitaxial growth on $\mathrm{Cu}(111)$ show that the adatom-vacancy pair yield increases from nearly 0 to 1 when the incident energy varies from 25 to $70 \mathrm{eV}^{23}$ Thus, although the defect-generation yields are not readily available for a $\mathrm{Ge}(001)$ surface bombarded by Ge, we expect, with increasing energy in PLD, more adatom-vacancy pairs will be created, thereby providing a higher concentration of mobile species on the surface, which, in turn, raises the vertical surface current acting to restore a rough surface to flatness. In their study of the relaxation of ion-etched $\mathrm{Ge}(001)$ surfaces, Chey et al. ${ }^{24}$ showed that the relaxation of rough $\mathrm{Ge}(001)$ surface is well described by the nonconserved step-mobility-limited model proposed by Uwaha. ${ }^{25}$ As step mobility is dependent on an atomic flux attaching to or detaching from a step, it is expected that having more mobile species will facilitate the relaxation process. ${ }^{26}$

As for the coarsening behavior of growth mounds, we first determine the mound separation, $d$ from the first maximum of the height-height correlation function of each image. Interest often lies in finding a so-called coarsening exponent assuming a powerlaw dependence of the mound separation on the film thickness, $d=d_{0}\langle h\rangle^{n}$, where $d_{0}$ is a prefactor, $<h>$ is the average film thickness, and $n$ is the coarsening exponent. The thickness-dependence of $d$ for MBE is shown in Fig. 3(a). A coarsening exponent of $0.17 \pm 0.02$ is obtained and is shown as a dashed line in Fig. 3(a). For PLD, the mound separation is sensitive to the deposition rate, which varies as discussed previously. To discern the coarsening behavior of growth mounds in PLD, we eliminate the effect of the deposition rate on the mound separation as follows. KMC studies on PLD have demonstrated that island distance in the sub-ML regime, $l_{c}$, is proportional to $I^{0.26}$, where $I$ is the instantaneous flux, ${ }^{9,27}$ and Politi and Villain ${ }^{2}$ showed that the lateral size of 
growth mounds induced by the ES barrier scales with $l_{c}{ }^{2} / l_{s}$, where the Schwoebel length $l_{s}$ is related to the strength of the ES barrier. ${ }^{28}$ A combination of these results yields $d \propto l_{c}^{2} / l_{s} \propto\left(I^{-0.26}\right)^{2}=I^{-0.52}$. We can replace $I$ with the deposition rate, $F$, because all the PLD films were grown at the same repetition rate, 20Hz. Furthermore, it was shown by KMC simulations that the coarsening exponent is a very weak function of the deposition rate, ${ }^{29}$ so we assume the deposition rate dependence of $d$ is contained in $d_{0}$, not in $n$. Normalization of $d$ is, therefore, accomplished by multiplying $d$ with $F^{0.52}$. Figure 3(b) shows normalized $d$, i.e. $d F^{0.52}$, vs. film thickness. The coarsening exponent of PLD-LE and PLD-HE is $0.33 \pm 0.03$, and $0.40 \pm 0.05$, respectively. MBE results normalized by the same way as in PLD are also shown for the purpose of a direct comparison. Several mechanisms have been proposed in order to explain coarsening of surface features appearing during epitaxial growth: deposition noise-assisted coarsening, ${ }^{30}$ coarsening driven by lateral surface current (either along step edges ${ }^{30,31}$ or via diffusion on the terrace bounding a mound ${ }^{16}$ ), or coarsening driven by capillaryinduced vertical surface current. ${ }^{32}$ These models assume a one-to-one correspondence between coarsening exponent and coarsening rate. Particularly, in the lateral surface current model, a current (either driven by the difference in step curvature or via random diffusion) flows into the region between mounds where a high density of kinks provides trapping sites. The coarsening rate is, then, dependent on the diffusional flux. Our result shows that the coarsening exponents fall in the order PLD-HE > PLD-LE > MBE. This is consistent with our speculation of the defect-mediated filling of gaps between growth mounds; incident particles of higher kinetic energy produce more mobile defects on the surface which accelerate the mechanism discussed above, resulting in a higher coarsening rate. Enhanced effective corner diffusion suggested earlier to rationalize the different morphology observed in PLD-HE can also be responsible for an increased coarsening rate as enhanced corner diffusion will result in an increase in lateral surface current. The scenario of a higher concentration of mobile species generated in PLD-HE leading to an enhancement of corner diffusion and a concomitant increase of lateral current is in agreement with the KMC simulation results by $\mathrm{Amar}^{16}$ that demonstrate an increased coarsening exponent in the presence of corner diffusion. Increased vertical downhill current, which is suggested as a possible mechanism in enhancing the epitaxial thickness in PLD, can also account for a higher coarsening exponent in PLD if vertical downhill current preferentially annihilates features with smaller $d$.

In summary, we have compared the evolution of morphology of $\mathrm{Ge}(001)$ homoepitaxial films grown by MBE and PLD under conditions nominally identical except for the different nature of the growth fluxes. Our results show that in MBE and PLD-LE, the morphology evolves in a similar way except that PLD films exhibit a higher areal mound density and an enhanced epitaxial thickness. PLD-HE yields a surface morphology different from the other two cases. The coarsening exponent is highest in PLD-HE, intermediate in PLD-LE and lowest in MBE. These observations are consistent with an increase in diffusional flux due to the higher yield of defect creation in PLD.

The authors acknowledge Prof. David Cahill at the University of Illinois for guidance in obtaining clean Ge surfaces. This research was supported by NSF DMR0306997. 


\section{REFERENCES}

* Present address: Department of Materials Science and Engineering, University of Pittsburgh, Pittsburgh, PA 15261-3848

** Present address: Glass Technology Center, PPG Inc., Hamar Twp., PA 15238

***email: maziz at harvard dot edu

${ }^{1}$ J. Villain, J. Phys. (Paris) 1, 19 (1991).

${ }^{2}$ P. Politi and J. Villain, Phys. Rev. B 54, 5114 (1996).

${ }^{3}$ N.E. Lee, G. Xue, and J.E. Greene, J. Appl. Phys. 80, 769 (1996).

${ }^{4}$ J.E. Van Nostrand, S.J. Chey, and D.G. Cahill, Phys. Rev. B 57, 12536 (1998).

${ }^{5}$ K.A. Bratland, Y.L. Foo, J. Soares, T. Spila, P. Desjardins, and J.E. Greene, Phys. Rev. $B$ 67, (2003).

${ }^{6}$ M.D. Johnson, C. Orme, A.W. Hunt, D. Graff, J. Sudijono, L.M. Sander, and B.G. Orr, Phys. Rev. Lett. 72, 116 (1994).

${ }^{7}$ K. Thurmer, R. Koch, M. Weber, and K.H. Rieder, Phys. Rev. Lett. 75, 1767 (1995).

${ }^{8}$ D.B. Chrisey and G.K. Hubler, "Pulsed Laser Deposition of Thin Films," (John Wiley, New York, 1994).

${ }^{9}$ B. Hinnemann, H. Hinrichsen, and D.E. Wolf, Phys. Rev. E 67, (2003).

${ }^{10}$ M.E. Taylor and H.A. Atwater, Appl. Surf. Sci. 127-129, 159 (1998).

${ }^{11}$ P. Ohresser, J. Shen, J. Barthel, M. Zheng, C.V. Mohan, M. Klaua, and J. Kirschner, Phys. Rev. B 59, 3696 (1999).

${ }^{12}$ J.M. Warrender, Ph.D. Thesis, Harvard University, 2004.

${ }^{13}$ J.A. Stroscio, D.T. Pierce, M.D. Stiles, A. Zangwill, and L.M. Sander, Phys. Rev. Lett. 75, 4246 (1995).

${ }^{14}$ X.J. Zhang, G. Xue, A. Agarwal, R. Tsu, M.A. Hasan, J.E. Greene, and A. Rockett, J . Vac. Sci. Technol., A 11, 2553 (1993).

${ }^{15}$ S.J. Chey, J. Van Nostrand, and D.G. Cahill, Phys. Rev. B 52, 16696 (1995).

${ }^{16}$ J.G. Amar, Phys. Rev. B 60, (1999).

${ }^{17}$ S. Schinzer, M. Kinne, M. Biehl, and W. Kinzel, Surf. Sci. 439, 191 (1999).

${ }^{18}$ In-situ reflection high energy electron diffraction measurements indicated the presence of a $2 \times 1$ surface reconstruction under all deposition conditions. Rapid diffusion directions on the $\{001\}$ surface should therefore be $<110>$ in contrast to $<100>$ on the bcc $\{001\}$ surface studied in Ref. 16 . Therefore, pyramidal mounds observed in our MBE and PLD-LE experiments with base edges oriented $45^{\circ}$ from $\left.<110\right\rangle$ are most directly comparable to mounds on bcc (001) with the edges oriented $45^{\circ}$ from $<100>$ observed in simulations with suppressed corner diffusion in Ref. 16.

${ }^{19}$ D.L. Smith, C.C. Chen, G.B. Anderson, and S.B. Hagstrom, Appl. Phys. Lett. 62, 570 (1993).

${ }^{20}$ N.E. Lee, G.A. Tomasch, and J.E. Greene, Appl. Phys. Lett. 65, 3236 (1994).

${ }^{21}$ J.A. Floro, B.K. Kellerman, E. Chason, S.T. Picraux, D.K. Brice, and K.M. Horn, J. Appl. Phys. 77, 2351 (1995).

${ }^{22}$ S.J. Chey and D.G. Cahill, Surf. Sci. 380, 377 (1997).

${ }^{23}$ J.M. Pomeroy, J. Jacobsen, C.C. Hill, B.H. Cooper, and J.P. Sethna, Phys. Rev. B 66, $235412(2002)$.

${ }^{24}$ S.J. Chey, J.E. Van Nostrand, and D.G. Cahill, Phys. Rev. Lett. 76, 3995 (1996).

${ }^{25}$ M. Uwaha, J. Phys. Soc. Japan 57, 1681 (1988). 
${ }^{26}$ However we cannot be certain of the dominance of downhill current over any uphill migration that may also be potentially induced by a higher concentration of mobile defects, see W. Zhu, F. Buatier de Mongeot, U. Valbusa, E.G. Wang, and Z. Zhang, Phys. Rev. Lett. 92, 106102 (2004).

${ }^{27}$ P.M. Lam, S.J. Liu, and C.H. Woo, Phys. Rev. B 66, 045408 (2002).

${ }^{28} \mathrm{It}$ is not guaranteed that the scaling of the mound separation by $l_{c}^{2} / l_{s}$ suggested by Politi and Villain can apply to PLD because their model system is for steady-state thermal deposition such as MBE. However, it is still plausible that in general the mound separation is proportional to $l_{c}$ weighted by a dimensionless parameter, $l_{c} / l_{s}$, which comes out naturally from the consideration of the ES barrier. In this sense, we may apply the scaling of mound separation to PLD in the presence of the ES barrier without introducing a significant error.

${ }^{29}$ J. Amar and F. Family, Phys. Rev. B 54, 14742 (1996).

${ }^{30}$ L.H. Tang, P. Smilauer, and D.D. Vvedensky, Euro. Phys. J. B 2, 409 (1998).

${ }^{31}$ T. Michely, M. Kalff, G. Comsa, M. Strobel, and K.H. Heinig, J. Phys.: Condens. Matter 14, 4177 (2002).

${ }^{32}$ M. Siegert, Phys. Rev. Lett. 81, 5481 (1998). 


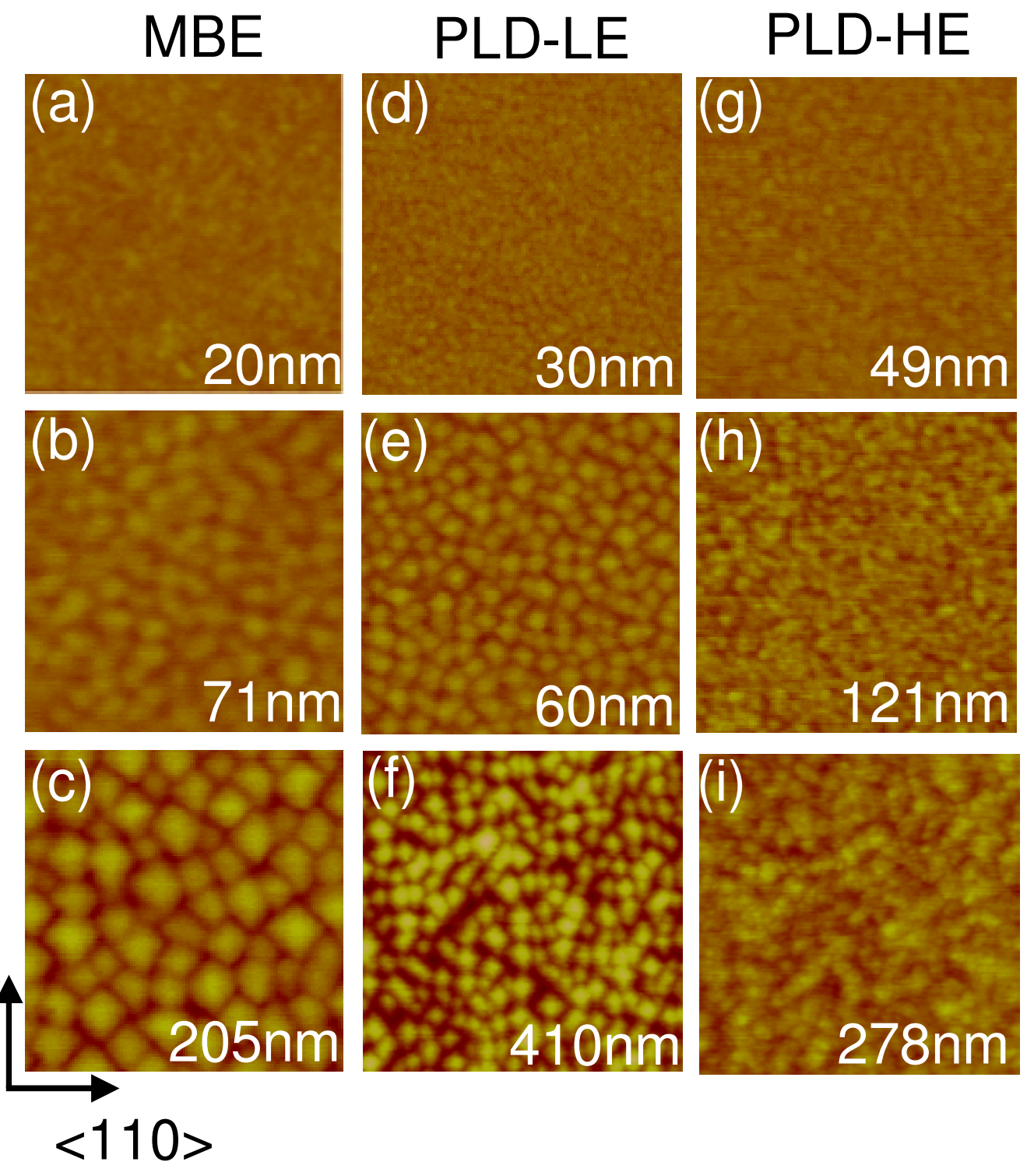

Fig. 1. AFM images of the films grown at $150{ }^{\circ} \mathrm{C}$ by MBE, (a) (c), PLD with low kinetic energy (PLD-LE), (d) (f), PLD with high kinetic energy (PLD-HE), (g) (i). The scan size and the grey scale of all images are $0.5 \times 0.5 \mu \mathrm{m}^{2}$ and $10 \mathrm{~nm}$, respectively. Thickness is shown in the right bottom corner of each image. 


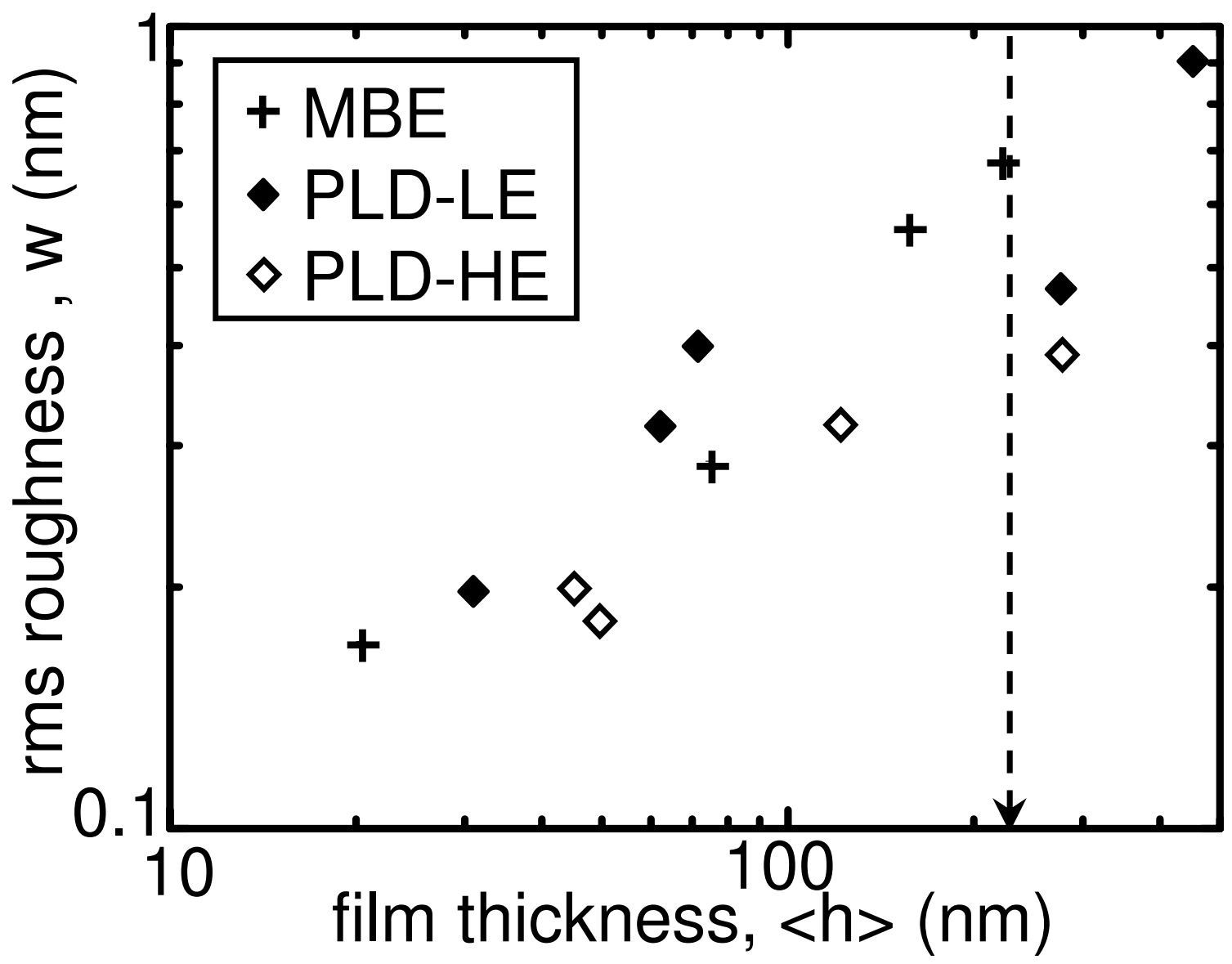

Fig. 2. Log-log plot of rms roughness vs film thickness. Vertical dashed line at $210 \mathrm{~nm}$ is added to indicate the epitaxial thickness of MBE film grown at $150{ }^{\circ} \mathrm{C}$. Films grown by MBE and PLD-LE have similar roughness except that epitaxial growth is extended beyond $210 \mathrm{~nm}$ for the case of PLD-LE. PLD-HE generates the smoothest films among all three cases. 


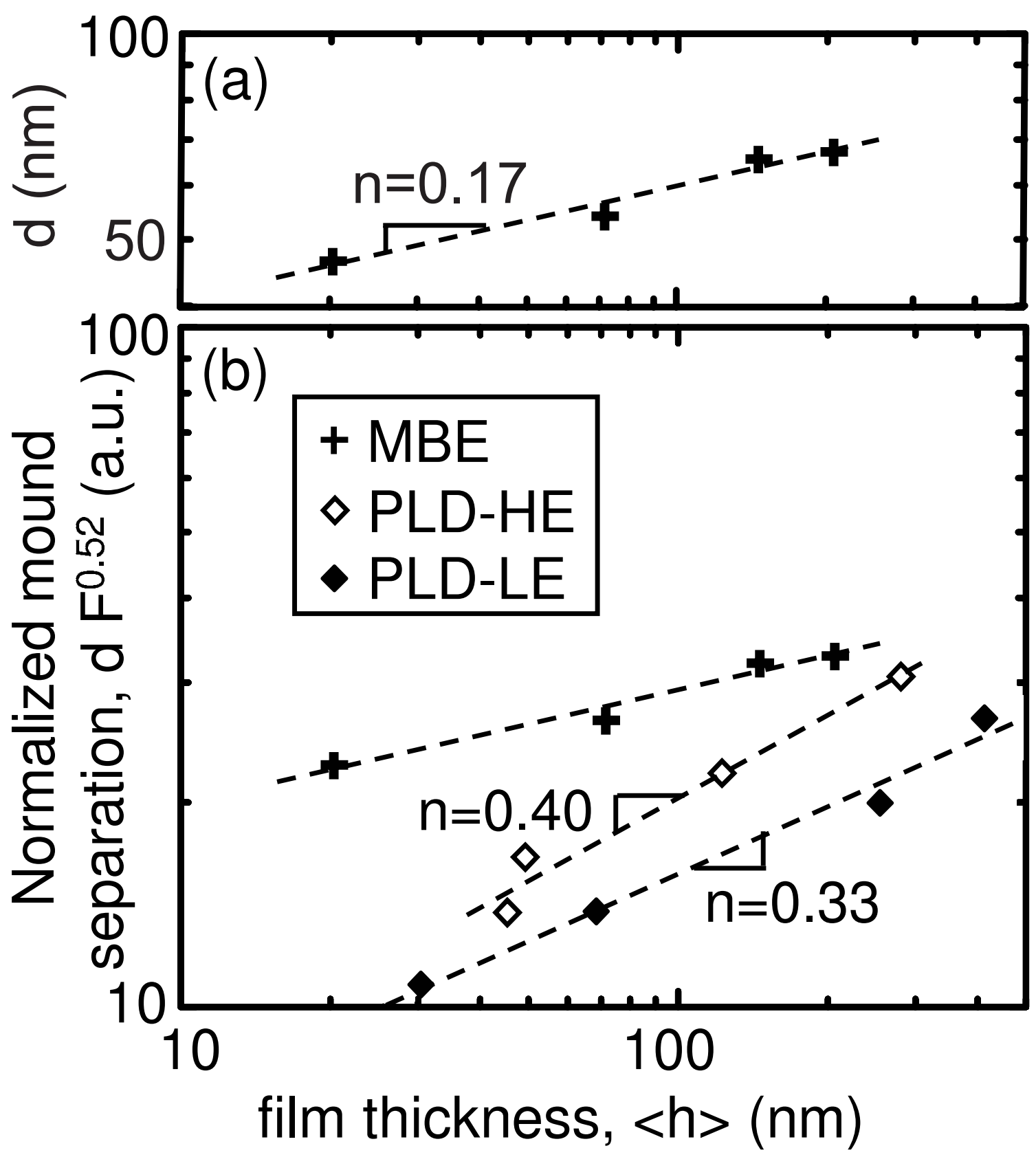

Fig. 3. Log-log plot of (a) mound separation, $d$, of MBE and (b) normalized mound separation, $d F^{0.52}$, of MBE, PLD-LE, and PLD-HE vs. film thickness. Normalization of $d$ by $F$ is discussed in text. Dashed line in each plot is a linear fit of data. The coarsening exponent is $0.17 \pm 0.02,0.33 \pm 0.03$, and $0.40 \pm 0.05$ for MBE, PLD-LE, and PLE-HE, respectively. 